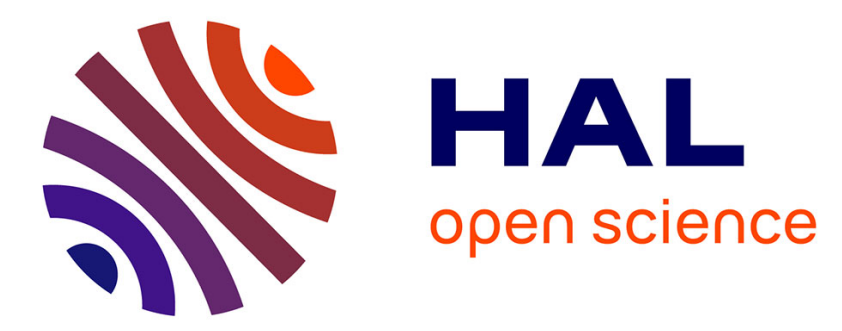

\title{
Arbitration for balancing control between the driver and ADAS systems in an automated vehicle: Survey and approach
}

\author{
Philippe Morignot, Joshué Pérez Rastelli, Fawzi Nashashbi
}

\section{To cite this version:}

Philippe Morignot, Joshué Pérez Rastelli, Fawzi Nashashbi. Arbitration for balancing control between the driver and ADAS systems in an automated vehicle: Survey and approach. 2014 IEEE Intelligent Vehicles Symposium (IV), Jun 2014, Dearborn, United States. pp.575 - 580, 10.1109/IVS.2014.6856577 . hal-01081302

\section{HAL Id: hal-01081302 https://hal.inria.fr/hal-01081302}

Submitted on 3 Dec 2014

HAL is a multi-disciplinary open access archive for the deposit and dissemination of scientific research documents, whether they are published or not. The documents may come from teaching and research institutions in France or abroad, or from public or private research centers.
L'archive ouverte pluridisciplinaire HAL, est destinée au dépôt et à la diffusion de documents scientifiques de niveau recherche, publiés ou non, émanant des établissements d'enseignement et de recherche français ou étrangers, des laboratoires publics ou privés. 


\title{
Arbitration for Balancing Control between the Driver and ADAS Systems in an Automated Vehicle: Survey and Approach
}

\author{
Philippe Morignot, Joshué Pérez Rastelli and Fawzi Nashashibi ${ }^{1}$
}

\begin{abstract}
Automated functions for real scenarios have been increasing in last years in the automotive industry. Many research contributions have been done in this field. However, other problems have come to the drivers: When should they (the drivers or the new automated systems) be able to take control of the vehicle? This question has not a simple answer; it depends on different conditions, such as: the environment, driver condition, vehicle capabilities, fault tolerance, among others. For this reason, in this work we will analyze the acceptability to the ADAS functions available in the market, and its relation with the different control actions. In this paper a survey on arbitration and control solutions in ADAS is presented. It will allow to create the basis for future development of a generic ADAS control (the lateral and longitudinal behavior), based on the integration of the application request, the driver behavior and driving conditions in the framework of the DESERVE project (DEvelopment platform for Safe and Efficient dRiVE ${ }^{1}$, a ARTEMIS project 2012-2105). The main aim of this work is to allow the development of a new generation of ADAS solutions where the control could be effectively shared between the vehicle and the driver. Different solutions of shared control have been analyzed. A first approach is proposed, based on the presented solutions.
\end{abstract}

\section{INTRODUCTION}

The aim of the Advanced Driver Assistance Systems (ADAS) is mainly related to help drivers in safety critical situations rather than to replace them. However, in recent years, many research advances have been done in this field, giving to understand that full autonomous driving is close to daily reality.

The number of automated functions for real scenarios have increased in the last few years in the automotive industry and research. However, other problems have appeared for drivers of such automated cars: When should the driver or the automated systems take control of the vehicle (since both cannot control an automated vehicle together at the same time due to potential conflicts)? In this work, we analyze the acceptability to the ADAS functions available in the market, and its relation to the different control actions, in order to propose a strategy to share control of the vehicle. For this, a survey on both arbitration and control solutions in ADAS is presented. This work will allow to create the basis for future development of a generic ADAS control (the lateral and longitudinal behavior of the vehicle), based on the integration of the application request, the driver behavior and driving conditions for the DESERVE project. The aim of DESERVE

\footnotetext{
This work was supported by INRIA and the ARTEMIS project DESERVE

${ }^{1}$ The authors are with the IMARA team, National Institute for Research in Computer Science and Control (INRIA), Domaine de Voluceau, BP 105, 78153 Le Chesnay, France (E-mail: firstname.lastname@inria.fr).

${ }^{1}$ http://www.deserve-project.eu/
}

is to design and more efficient architecture process in order to come to a reduction in costs of components for the standardisation of ADAS functions in future vehicles.

The main goal of this work is to allow the development of a new generation of ADAS solutions where control could actually be shared between the automated vehicle and the driver. In this paper, we analyze various solutions of shared control and will deepen these concepts for arbitration in future work.

This paper is organized as follows: Section II performs a survey on the notion of arbitration for automated systems (automation layers, arbitration, driver monitoring and control passing). A description of key factors for vehicle control applications (based on ADAS) in the market is presented in section III. A proposal based on Fuzzy Logic for arbitration controllers is presented in section IV. Finally, the last section sums up our contribution.

\section{A SURVEY ON ARBITRATION}

The domain of ITS (Intelligent Transportation Systems) has produced success stories over the years such as Cybercars (90s), the DARPA Grand Challenge won by the Junior car (2007), international projects as VIAV - : international travel with autonomous vehicles from Parma to Shanghai - by VisLab (2010), the Grand Cooperative Driving Challenge (GCDC-2011), to cite a few. In this view, the driver of a car / truck is replaced by a computerized automation, which perceives its environment through sensors (Radars, cameras, GPS, Inertial Measure Units and odometry), and completely drives the vehicle in urban, sub urban or regional traffic.

However, nowadays daily computerized vehicles cannot ensure full autonomy in all situations, i.e., co driving still is necessary to ensure the safety in the overtaking maneuver when another is coming in the opposite road in two-way roads-[1]. As such, a driver and an automated device (the co system) must coexist inside a unique vehicle, one assisting the other when required by the situation at hand. This coexistence involves an intermediate concept to properly balance control in all situations, called automation layers or autonomy levels. This also involves arbitration, as the concepts and mechanisms needed to produce a unique motion of the vehicle given the two decision makers, the automation and the driver, which must harmonize their respective decisions (see Fig. 2). We propose below a survey on these notions: We first review autlomation layers as the backbone notion towards arbitration, then propose a theoretical framework over arbitration, and finally cover related concepts 
(monitoring the driver as part of arbitration and passing control between the driver and the co-system).

\section{A. Automation layers}

Full automation of mechanical devices is a goal of both Robotics and A.I. for decades. The dream of an autonomous robot that would act without needing any supervision or commands from a human has been shared by members of the robotic and A.I. communities since the early stages of these scientific domains. The same aim inhabits the ITS community since the 90 s as well (a vehicle that would autonomously drive in a daily traffic). For example, in aeronautics, drones (UAVs) can operate with little intervention from a pilot: observation war drones are joystick guided by an on ground pilot [2]. Similarly pilots of commercial aircraft sometimes can avoid any intervention on the control of the aircraft (Flight Management Systems). But, even if aircrafts are close to full autonomy, safety requires that a pilot be in the cockpit, for checking the automation and reacting when automation fails or when an unexpected event occurs which was not planned by the automation designers of the aircraft.

Regarding safety, embedding source code on commercial aircraft must comply with recommendations of dedicated norms and standards, e.g., DO 178B and followers, to obtain authorization from public authorities to make source code actually fly on a commercial aircraft with passengers life depending on it [2].

This problem of automation of robotic devices has been identified for long, and current research activities tend to go beyond the Boolean description full autonomy or not full autonomy" (e.g., fully manual) by tightening the cooperation between a driver (or pilot) of the physical device and the automated physical mechanism itself. Typically, a spectrum of automation layers or levels between the two extreme positions above is defined, which describes to which extent the mechanical robot can coexist with a human driver or (eventually remote) pilot, the result of which being the actual motion of the vehicle, terrestrial or aerial. However, if there is a global consensus on the extreme layers fully manual and fully autonomous, authors disagree on the number and nature of intermediate layers in between.

The level of automation can be defined as a combination of tasks delegated at some level of abstraction with some authority and resources delegated with some level of authority to be used to perform that task. The level of automation in a human-machine system increases whenever that the level of abstraction, aggregation or authority increases [3].

For example, in aeronautics, a spectrum of 11 automation layers has been proposed for unmanned aerial vehicles UAVs [4], from the lowest autonomy level Remotely piloted vehicle (level 0) up to the upmost layer Fully Autonomous (level 10) through intermediate layers such as Robust Response to Anticipated Faults/events (level 3 on a spectrum from levels 0 to 10). Similarly, 10 layers have been proposed to describe the cooperation between a human and any automated mechanical device, not necessarily a UAV or an automated car [5]. In this view, the lowest level is stated as The computer offers no assistance: human must take all decisions and actions while the upmost layer is stated as The computer decides everything, acts autonomously, ignoring the human through intermediate layers such as Allows human a restricted time to veto before automatic execution (level 6 on a spectrum from levels 1 to 10 , see Figure 1).

10. The computer decides everything, acts
autonomously, ignoring the human.
9. Informs the human only if it, the computer,
decides to
8. Informs the human only if asked
7. Executes automatically, and then necessarily
informs the human
6. Allows the human a restricted time to veto
before automatic execution.
5. Executes that suggestion only the human
approves it.
4. Suggests one alternative
3. Narrows the selection down to a few
2. The computer offers a complete set of
decision/action alternatives
1. The computer offers no assistance: human
must take all decisions and actions

Fig. 1. Levels of automation of decision and action selection (excerpt from [5]).

In the ITS domain, 21 automation layers have been proposed to give a frame to the co driving activity [6], with an ontological representation. In this ontology, the lowest layer is Fully manual to the upmost layer Cooperative parking through intermediate layers such as Lane following and Obstacle avoidance.

Some authors also define more precisely these automation layers by stating to which part of the automation process these layers refer to. Hence, not only a one dimensional spectrum of layers is defined, but also a two dimensional matrix of automation layers is defined, since there is a horizontal dimension to the automation layers above. For example, Clough [4] defines such a horizontal spectrum with: (1) perception / situation awareness (observe); (2) analysis / Coordination (orient); (3) Decision making (decide); And (4) capability (act). The 11 automation layers of [4] can be expressed for each one of these 3 other elements along this horizontal dimension.

Parasuraman et al. [5] define a close horizontal dimension, to cover the chain of processing with human intervention, based on the processing flow from inputs to outputs of the automated mechanism:

- Information acquisition: "In the sensing process, this step involves mechanically moving sensors in order to scan and observe, with highlighting and filtering as goals".

- Information analysis: "This can be for example extrapolation of data over time (prediction). For example, 
predictor displays have been designed in the cockpit of aircraft pilots that show the projected future course of another aircraft in the neighboring space".

- Decision and action selection: See Figure 1 for the automation layers of this horizontal element.

- Action implementation: This refers to the actual execution of the action choice.

Despite the disagreement of authors regarding the number and nature of automation layers except for the upmost and lowest layers), there surely is an agreement on the existence of a spectrum of layers, sometimes in 2 dimensions. Parasuraman et al. [5] is one of the most cited authors to this regards (over 1000 hits on Citeseer), and their spectrum and decomposition seem to be adopted by other authors in the ITS domain (e.g., see Schieben et al. [7]).

\section{B. Arbitration}

Arbitration has been very little studied by previous authors, so we present the work we are aware of.

Arbitration in ITS comes from the fact that co driving involves two decision makers, the driver and the computerized automation, inside a unique vehicle, and that there must be a unique path and trajectory followed by this vehicle, despite these two decision makers (see Figure 2). The cosystem may have reached some level of automation on the previous spectra (see previous section), first made possible by the advance in ADAS systems towards more automation (see section III), and second due to dynamic update of the automation level of the ADAS system which must stay compatible with the autonomy level of the driver.

For example, at an intersection, what if the driver wants to go to the right and the automation "wants" to go to the left? (i.e., on Fig 2, the action ("act" on the bottom) sent by the human is incompatible with the action ("act" on the top) sent by the computer). Negotiation is here enforced, which might unfold under severe conditions in time critical situations.

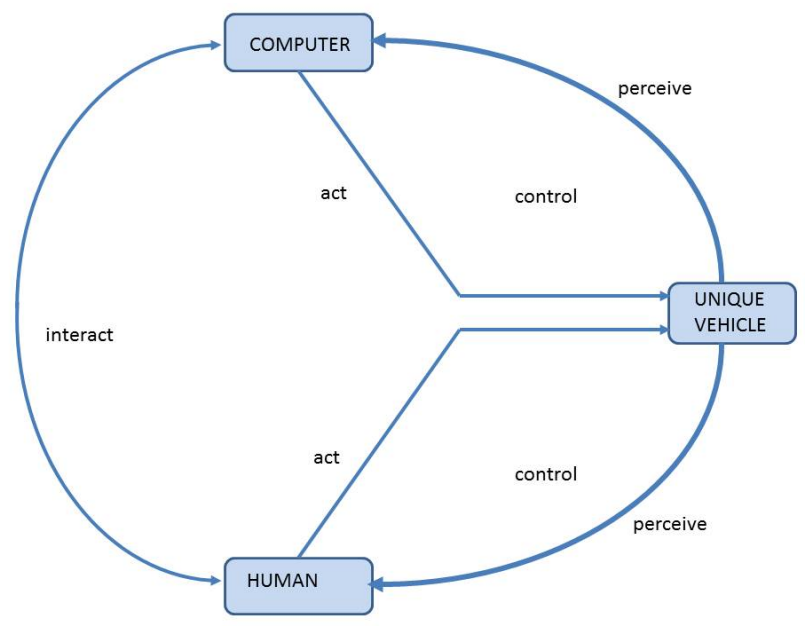

Fig. 2. Cooperative control between a pilot and a co pilot, human or automated (excerpt from [8]).

The previous section defines automation layers in a static way, whereas these layers can be used dynamically. That is, the level of autonomy of the co-system should adapt to the what the ADAS system is capable to bring to driving, and to what the driver state allows. If the ADAS systems is no advanced enough, no co driving is possible (the automation layer 0 is reached only, according to the spectra of the previous section).

For example, given a level of tiredness of the driver, supposed awake and fully aware of his means, the automation level of the vehicle can be set to low, i.e., levels 0 or 1 on the spectra of the previous paragraph. But if the driver loses his resources and falls asleep, then the level of automation of the vehicle should increase, as a response to the collapsed state of the driver. This is a control change in the automation layer.

To take another example opposite to the previous one, suppose that the automated car drives autonomously on a highway under clear situation, and that an imminent danger suddenly comes into the situation. The automated car should use its means to warn the driver to take back control to deal with this danger, if there is enough time for this procedure to be executed. If time is too critical for waiting for the driver acknowledgement of control change, the automated car should act alone (e.g., braking, coming back to the lane, changing lane). The level of assistance of the car might change depending on the drivers state. With a varying level of automation of the automated vehicle, control might smoothly flow from the driver to the automated car and from the automated car to the driver (see section II-D).

In other words, arbitration takes as input the imminence of danger, the software available onto the automated vehicle, the state of its sensors, the environment state and the driver state, and produces as output an automation level of the automated vehicle.

In order to organize the concepts involved in arbitration and control flow between a driver and an automated vehicle, Flemisch et al. [8] propose four concepts in the ITS domain: Ability, considered as "the possession of means or skill to do something"; Authority, seen as "the power or right to give orders, make decisions and enforce obedience"; Responsibility, defined as "a moral obligation to behave correctly"; and Control, seen as "having the power to influence the course of events".

These concepts hold relations for ITS, as depicted in Figure 3. These common notions from daily life can be rephrased for ITS, by understanding the "means" of ability as the interface between the driver and the automated car; "orders" as using the interface between the driver and the car; "behaving correctly" as following its path and avoiding danger; And "influencing the course of events" by choosing the trajectory of the vehicle. With these equivalences in mind, the preceding four concepts seem to fit into shared and cooperative control of an automated vehicle.

The relationships among these four concepts (see Figure 3) can be explained by the following: "Control cannot be successful without enough ability. Similarly, the appropriate authority is needed to be allowed to control. A certain responsibility might motivate the actor to execute control. 


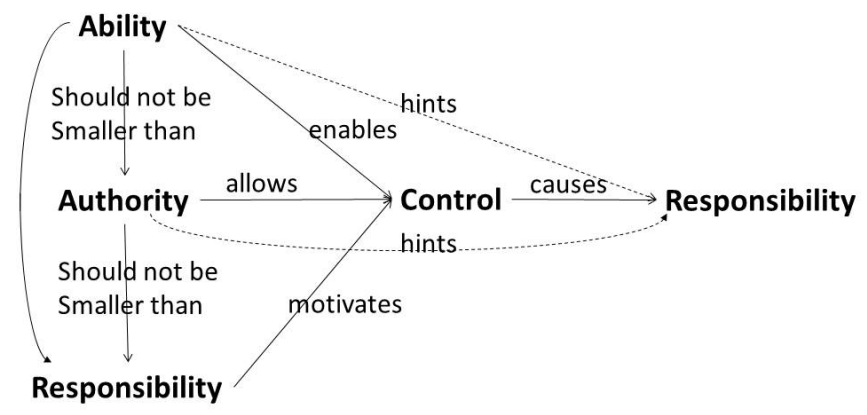

Fig. 3. Relations between ability, authority, control and responsibility (excerpt from [8]).

The portion of control for which responsibility is assigned should be less or equal to the portion of control for which authority is granted and ability is available, since responsibility without sufficient authority and ability would not be fair." [8]

\section{Driver monitoring}

Driver monitoring [9] is another aspect of arbitration. It can be considered as the surveillance of the state of the driver by the automated vehicle, in order to determine whether he or she is capable of fulfilling his/her tasks in a robust and optimal way. This is especially important if the level of automation of the vehicle is low, i.e., the demand on the driver is high. According to the previous vocabulary, the control authority has to decide sometimes quickly whether control has to be moved higher towards upper automation layers and lower for the driver.

Rauch et al. [9] have investigated driver monitoring inside the EU-funded project HAVEit, by using a driving simulator while observing many drivers driving under different conditions. This approach requires that the automated vehicle is capable of sensing the driver and his state: this is performed through a camera on the front board of the vehicle, and pointing at the drivers face. A real-time algorithm is used to detect whether the driver looks on/off the road. More precisely, this detection is based on the driver's eyelid blinking pattern (blink duration, closing and opening durations, blink amplitude), eye movements and gaze direction, all as direct measures. Indirect measures of the driver's state involve observing the frequency of the lateral deviation of the vehicle on its lane (fast steering velocity, frequency of the steering wheel velocity, lane crossings). Both direct and indirect measures are correlated to determine the state of the driver on the following spectrum (excerpt from [9]):

- "Asleep / unresponsive: Complete collapse, full breakdown of performances".
- "Sleepy: resource exhaustion, heavy performance impairments".

- "Drowsy: high effort investment, need for compensation".

- "Slightly drowsy / low vigilance: start of effort investment, without need for compensation".

- "Awake: full resources available.

For the practical experiments with a driving simulator performed by Rauch et al. [9], only three states were detected : asleep, drowsy and awake. An interesting correlation between the measures above and these driver's states was observed. Typically, the following measures increase as a function of the sleepy state of the driver: standard deviation of the lateral position, number of lane crossings, steering wheel velocity and percentage of occurrence of steering wheel motion of more than 50 degrees per second. That is, the more sleepy the driver, the more he deviates laterally, the more number of lanes he crosses and the more he fixes his lateral position by strong motion on the steering wheel.

\section{Control passing}

Control passing involves switch of control from the driver to the automated vehicle, or vice versa, at automation or driver's request. Experiments as a Human Factor study on this topic have been performed [7] inside the EU-funded project HAVEit. Using a vehicle simulator, these authors study how control flows from the vehicle to the driver, and back, depending on the situation (for simplicity of measures, only 3 layers of automation are defined for the vehicle, low, medium and high): 8 persons are asked to drive in a driving simulator, simulating an automated car with the automation layers "driver assisted" (lane keeping assistance), "semi automated" (longitudinal control with advanced ACC) and "highly automated" (hands off). These authors then draw recommendations on the design of the interface of a vehicle (e.g., location of the autonomy level switch rod, information displayed to the driver), hence improving the usability of a driving environment by drivers.

\section{ADAS APPLICATIONS: A CONTROL PERSPECTIVE}

Modern ADAS functions help drivers in different tasks or situations in the driving process: the more advanced ADAS functions are, the higher automation layer they reach (see discussion of the previous section), the less load on the driver.

Some studies show that nowadays there are many distractions for drivers, causing multiple task overloads (e.g. GPS, panel recognition, security alarms, among others) . One of the most common causes of driver distraction is the monotonous driving. It causes mental underload, which decreases the vigilance on the route and then it generates dangerous situations. Moreover, the stress, mental overload and highly difficult situations are important factors to be considered in the definition of the interaction between driver and fully autonomous functions. The project HAVEit has obtained a pragmatic approach for the levels of assistance and automation in vehicles. These are: Driver only, Driver 
assisted, Semi-automated, Highly automated and Fully automated, as a simplification of the automation layers of Figure 1 for driving applications.

\section{A. Key factors for vehicle control in the market}

The Advanced Driver Assistance Systems are one of the objectives of the ITS along with intelligent infrastructure and autonomous driving developments. Recently, the ADAS are more accepted by consumers. In [10] a study of awareness and interest of the Driver Assistance Systems and Active Safety Features on vehicle is described considering: driver warning, assistance and map enabled systems, HMI preferences and user-friendliness of current safety systems, perceived benefits of integration with chassis and powertrain and other future safety systems and technologies. Some other motivations to the development and improvement of these systems are:

- Legislation: Different initiatives like European New Car Assessment Programme (Euro NCAP) have been developed recently. The most relevant advanced safety technologies are rewarded for the Euro NCAP, such as: Blind Spot Monitoring, Lane Support Systems and Emergency braking, among others [11].

- Cost: Studies show that customers are willing to pay more for avoidance systems than for other ADAS in their vehicles [10].

- Market indicator: Some of them show that the ADAS will have a high influence on market in few years [10].

- Safety: on board Electronic systems have become critical to the functioning of modern automobiles, as mentioned by the national research council report [12].

The individual functions of the ADAS are designed from the beginning in such a way that they operate within a common environment. Different ADAS functions will not simply live together, nevertheless coexist and deeply cooperate by providing their assistance to the drivers simultaneously. Some of the technology availability is:

- Interconnections: Electronic systems are being interconnected with one another and with devices and networks external to the vehicle to provide their desired functions [12].

- Fusion: New vehicle capabilities have to be adapted to the human behavior in the driving process, and electronics, perception system information and HMI inputs become relevant in order to handle the best decision in each situation.

- New infrastructures: A recent study report from the European Commission explains the need of vehicle and infrastructure systems designed for Automated Driving [13].

- Sensor technologies: They are becoming more sophisticated and varied, especially to support the functionality of many new convenience, comfort, and safety-related electronic systems.

\section{FUZZY LOGIC APPROACH FOR SHARING CONTROL OF} A VEHICLE

In this section, we propose an approach on arbitration based on fuzzy logic.

Decision making and intention detection concepts are usually based in the combination and evaluation of measurable parameters to infer a probability value. Based on the information that comes from different perception systems, it is possible to define fuzzy control parameters. Emulating human driving, Zadeh [14] has proposed several examples where fuzzy control could be applied. Among them, the decision marking in autonomous driving process is an interesting field to apply fuzzy logic using human driver experience as expert knowledge [15].

This cognitive process will result in the selection of a course of action among several alternative scenarios (e.g., if the driver should take the control of the pedal in ACC manoeuvre while tired). Every decision making should adapted to each scenario defined in the framework of DESERVE project. Based on these previous works a first approach based on fuzzy logic techniques is presented in this work.

The rule base interprets the input variables in a process termed inference. This approach allows fuzzy rules to be written as sentences in an almost natural language, as in [15]. The sentences used are of the following form: IF... THEN..., for instance:

IF driver fatigue level is high AND TTC is low THEN Fully control level is HIGH

IF driver fatigue level is low AND TTC is high THEN Fully control level is LOW ...

Where TTC is the Time To Collision, and the Fully control level is output defined as singleton value [14].

\section{A. Module Considerations and inputs}

This module will use the information from the Driver monitoring, Frontal Object Perception, Vulnerable Road User, Lane recognition and vehicle trajectory calculation (based on the demonstrators available in the project [16]). An estimation of the driver stage (tired, sleepy, attentive, angry, etc) is sent to Arbitration module, based on interior camera and biomedical sensors.

This module can consider the information from multiple sensors, in order to establish a risk level in each scenario. This information will be used to determine if the driver is capable to handle a danger situation.

The output will determine the action to be taken by the driver. Two main decision makers are considered: when does the driver take the control, or when does the automated systems take control. This module can consider the Trajectory planning, Driver stage and Risk Management information. The output determines who should drive the vehicle.

\section{CONCLUSION ANF FUTURE WORK}

This paper presents (1) a survey on arbitration and control solutions for ADAS, based on the ADAS solutions available in the market, and considering the functional requirements 
described in the framework of the DESERVE project; And (2) a first approach towards arbitration using fuzzy logic.

Different points of view have been considered in the solution of the arbitration problem, despite the lack of agreement on the automation layers nature. The estimation of the driver stage is one of the most important parameters to be considered for sharing the vehicle control. For this reason, it is interesting to notice the contribution to the perception layer, especially in the driver monitoring approach.

In the future works of our research, an estimation of the stage of the driver will be used to arbitrate if the vehicle can be controlled by him or the autonomous system. Since the driver is legally responsible for operating the car in its environment, in our approach the last responsibility will be for the drivers. The behavior of a human driver can be emulated with Fuzzy Logic techniques. The next step is to design the controllers considering the variables to be used in the demonstrator. The inputs and output signals that will be used in our approach, as well as the rule base, they will based on drivers experience in different scenarios.

We will focus on the arbitration, to determine (using some perception information) when the driver should take the control of the vehicle, and which situations are more dangerous (risk management). This implementation will be done in a first stage in simulation, and then it will implement in real demonstrators.

\section{ACKNOWLEDGMENT}

Authors wants to thank to the ARTEMIS project DESERVE for its support in the development of this work.

\section{REFERENCES}

[1] J. Pérez, V. Milanes, E. Onieva, J. Godoy, and J. y Alonso, "Longitudinal fuzzy control for autonomous overtaking," in IEEE International Conference on Mechatronics (ICM'11), 2011, pp. 15 - 21.

[2] R. E. Berk, "An analysis of current guidance in the certification of airborne software," Massachusetts Institute of Technology, Tech. Rep., 2009.

[3] C. Miller, "Using delegation as an architecture for adaptive automation," Airforce, Tech. Rep. AFRL-HE-WP-TP-2005-0029, 2005.

[4] B. Clough, "Metrics, schmetrics! how the heck do you determine a uavs autonomy anyway?" in Performance Metrics for Intelligent Systems Workshop, Gaithersburg, MA, USA, 2002.

[5] R. Parasumaran, T. B. Sheridan, and C. D. Wickens, "A model for types and levels of human interaction with automation," IEEE Transactions on Systems, Man and Cybernetics - Part A: Systems and Humans, vol. 30, no. 3, pp. 286-297, May 2000.

[6] E. Pollard, P. Morignot, and F. Nashashibi, "An ontology-based model to determine the automation level of an automated vehicle for codriving," in 16th International Conference on Information Fusion (FUSION13), Istanbul, Turkey, July 2013.

[7] A. Schieben, G. Temme, F. Köster, and F. Flemish, "How to interact with a highly automated vehicle generic interaction design schemes and test results of a usability assessment," in Human factor Human Centered Automation, D. de Waard, N. Gerard, L. Onnasch, R. Wiczorek, and D. Manzey, Eds. Shaker, 2011, pp. 49-56.

[8] F. Flemisch, M. Heesen, T. Hesse, J. Kelsch, A. Schieben, and J. Beller, "Towards a dynamic balance between humans and automation: authority, ability, responsibility and control in shared and cooperative control situations," in Cognitive Technology Work, 2012, vol. 14 , pp. $3-18$.

[9] N. Rauch, A. Kaussner, H.-P. Krüger, S. Boverie, and F. Flemisch, "The importance of driver state assessment within highly automated vehicles," in 16th ITS World Congress and Exhibition on Intelligent Transport Systems and Services, Stockholm, Sweden, 2009.
[10] 2009 European Consumers Desirability and Willingness to Pay for Advanced Safety and Driver Assistance Systems (Frost \& Sullivan).

[11] Euro NCAP, Moving forward, 2010-1015 Strategic Roadmap, December 2009.

[12] Transportation Research Board, special report 308: the safety Promise and challenge of Automotive electronics, Insights from unintended acceleration, National Research Council of the national academies.

[13] European Commission, Definition of necessary vehicle and infrastructure systems for Automated Driving: Study Report, SMART 2010/0064.

[14] L. A. Zadeh, "Outline of a new approach to the analysis of complex systems and decision processes," IEEE Trans. on Systems, Man, and Cybernetics, vol. SMC-3, pp. 28-44, 1973.

[15] J. Pérez, V. Milanes, E. Onieva, J. Godoy, and J. Alonso, "Longitudinal fuzzy control for autonomous overtaking," in Proc. IEEE International Conference on Mechatronics ICM 2011, 14-17 April 2011, pp. 1-5.

[16] D24.1 Vehicle Control Solutions, WP Arnitration and control, DESERVE project, 2013. 